\title{
Kassenfusion
}

\section{Barmer GEK und Deutsche BKK planen gemeinsame Zukunft}

Deutschlands zweitgrößte Krankenkasse, die Barmer GEK, und die Deutsche BKK haben beschlossen, künftig gemeinsam anzutreten, und bereiten eine Fusion der beiden Kassen vor. Die neue Krankenkasse nimmt dann zum 1. Januar 2017 ihre Arbeit auf. Beide Unternehmen versprechen sich von der Vereinigung insbesondere Vorteile beim Service für die Versicherten und in der Optimierung der Firmenkundenbetreuung. Derzeit hat die Barmer GEK mehr als 8,5 Millionen Versicherte, die Deutsche BKK etwa 1,1 Millionen Versicherte. Die neue Krankenkasse wird über eines der dichtesten Geschäftsstellennetze in der gesetzlichen Krankenversicherung verfügen.

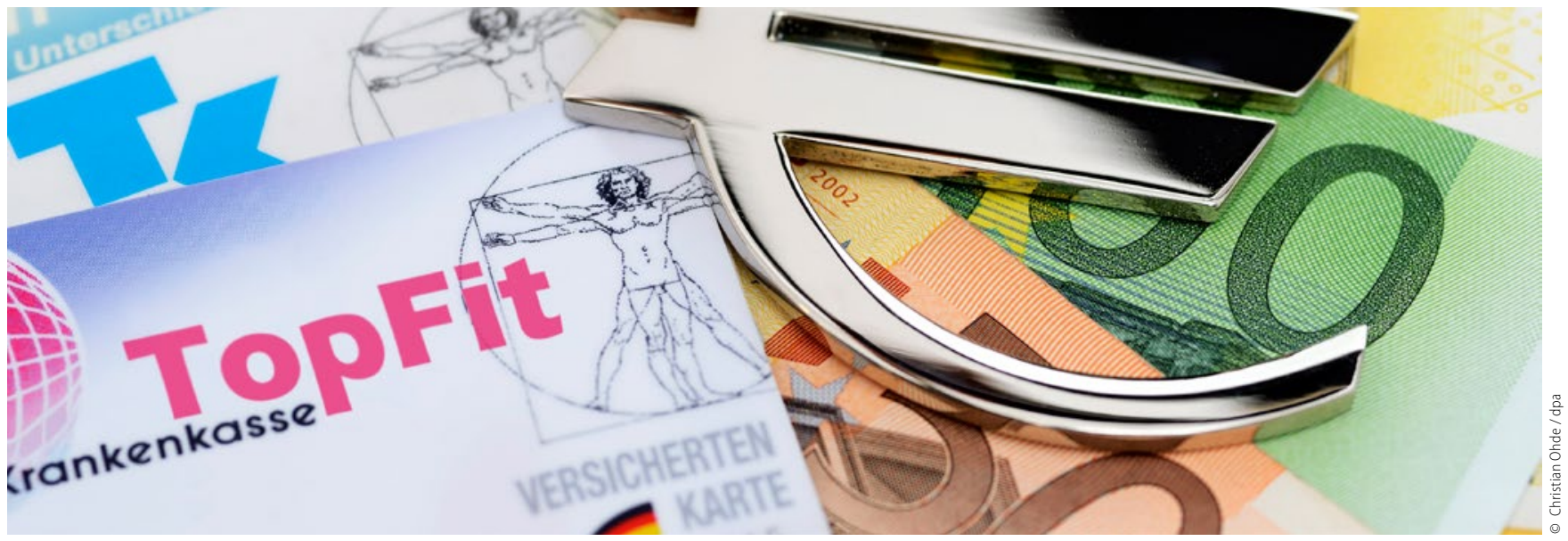

Gesetzliche Krankenversicherung

\section{Gesundheitsministerium legt Zusatzbeitrag für 2016 fest}

Nun ist es offiziell: Nach Angaben des Bundesgesundheitsministeriums (BMG) wird der durchschnittliche Zusatzbeitrag in der gesetzlichen Krankenversicherung (GKV) im nächsten Jahr um 0,2 Prozentpunkte auf 1,1 Prozent ansteigen. Grundlage ist die Prognose des Schätzerkreises zur Einnahmen- und Ausgabenentwicklung der GKV, der aus Experten des BMG, des Bun-

\section{Erstes Erklärvideo Implantate}

proDente veröffentlicht ein Erklärvideo zum Thema Implantate. Erstmals rückt Servatius Sauberzahn in den Mittelpunkt eines Films.

Der „Zahnarzt“ Servatius Sauberzahn ist ein fiktiver Charakter, der bislang vor allem Informationen in den sozialen $\mathrm{Me}$ dien verbreitet.

„Ein Erklärvideo bietet sich bei komplexen Themen an, da es Informationen leicht verständlich transportiert", kommentiert Dirk Kropp, Geschäftsführer proDente, die Entscheidung für das Filmformat. Mit den illustrierten Animationen kann der Patient Abläufe sehen, die ihm bei der realen Filmaufnahme einer Operation verborgen bleiben. Zugleich ist die Darstellung des chirurgischen Eingriffs für sensible Zuschauer erträglicher. desversicherungsamtes und des GKV-Spitzenverbandes besteht. Wie hoch der individuelle Zusatzbeitrag für die Arbeitnehmer ausfällt, kann jede Kasse selbst festlegen. Erhebt eine Kasse einen Zusatzbeitrag über den vom BMG festgelegten Durchschnitt, muss sie ihre Versicherten über die Wechselmöglichkeiten zu einer günstigeren Kasse aufklären.

cas

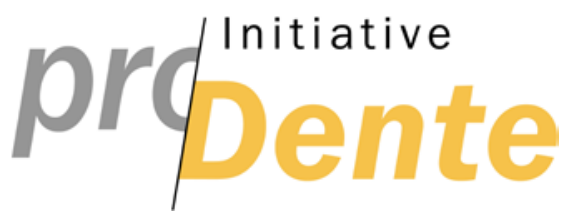

\section{Implantate - Schritt für Schritt erklärt}

Thema des ersten Erklärvideos von proDente ist Zahnersatz auf einer künstliche Zahnwurzel - einem Implantat. Schritt für Schritt erklärt Servatius Sauberzahn den Vorgang. Der Link zum Fimbereich: http://bit.ly/videos-filme.

\section{proDente-App jetzt im Google Playstore}

Aktuelle Informationen über die Aktionen der Initiative und nützliche Tipps gibt es jetzt auch per App. Unter http://bit. ly/playstore-proDente kann die Anwendung für Smartphones und Tablets mit dem Betriebssystem Android heruntergeladen werden. 